\title{
Bacterial diversity and its relationship to growth performance of broilers
}

\author{
Yeonji Bae, Bonsang Koo, Seungbaek Lee, Jongsuk Mo, Kwanghyun Oh, In Pil Mo* \\ Veterinary Medical Center and College of Veterinary Medicine, Chungbuk National University, Cheongju 28644, Korea
}

(Received: June 7, 2017; Revised: August 18, 2017; Accepted: August 22, 2017)

\begin{abstract}
The microbial community is known to have a key role during the rearing period of broilers. In this study, gut microbial composition and diversity were examined to evaluate the relationships between these factors and broiler growth performance. By applying 454-pyrosequencing of the V1-V3 regions of bacterial 16S rRNA genes, six fecal samples from four- and 28-day-old chickens from three broiler farms and 24 intestinal samples of broilers with heavy and light body weights were analyzed. Microbial composition assessment revealed Firmicutes to be the most prevalent phylum at farm A, while Proteobacteria were predominant at farms B and C. Fecal microbial richness and diversity indices gradually increased from four to 28 days at all three farms. Microbial diversity assessment revealed that small intestine microbial diversity was lower in heavy birds than in light birds. In light birds, the Firmicutes proportion was lower than that in heavy birds. In conclusion, each broiler farm revealed a specific microbial profile which varied with the age of the birds. The microbial communities appeared to affect growth performance; therefore, gut microbial profiles can be utilized to monitor growth performance at broiler farms.
\end{abstract}

Keywords: broiler, growth performance, microbial community, pyrosequencing, 16S rRNA gene

\section{Introduction}

The gut microbiota in chickens consists of diverse prokaryotic and eukaryotic microbes, which live synergistically in their host [38]. The gut microflora plays a key role in maintaining a conditioned status by the competitive exclusion of pathogens. It is also related with a number of reactions, such as the metabolization of nutrients, the development of immune responses, and the regulation of both health and diseases [25, 34]. In poultry, several studies have suggested that there is significant diversity in bacterial populations among different parts of the gastrointestinal tract (GIT) [2, 22], and that the microbial profiles of the GIT are connected to the growth and health of flocks [26, 29].

Gut health can be affected by various factors, such as the host, feed, litter, farm facilities, and environment [12]. Disturbances in the balance of the gut microbiota subsequently impair feed efficiency and growth performance [39]. The growth performance of broilers is one of the most critical concerns for producers. Several parameters such as the feed conversion ratio (FCR), body weight at market age, and livability were included in the elements of performance indices and used to determine the body performance of broiler flocks. Antibiotic growth promoters in food animal production have been used to enhance the growth performance and the health status of animals since the mid-1940s [18]. However, antibiotics-free programs have been established worldwide and these have induced a negative impact on body weight gain and frequent occurrences of bacterial enteritis in broiler farms [9].

Bacterial enteritis can cause economic losses, increased mortality, the disturbance of animal welfare, and risks the contamination of products for human consumption [32]. The presence of specific bacterial species and a variety of environmental factors were involved in outbreaks of enteritis among broilers. These extrinsic and intrinsic factors also include medication exposure, diet, litter management, housing type, biosecurity, flock age, genetic elements mediating drug resistance, and the gut microbial community $[1,10]$. Until recently, a number of previous studies on poultry bacterial populations have relied on conventional culture methods to identify infectious agents and enumerate bacterial species [14]. However, such labor-intensive and time-consuming cultivation basedmethods have limitations in their ability to provide comprehensive observations of chicken's gut microbiota.

The metagenomic approach, enhanced by high-throughput sequencing technologies, has begun to fulfill expectations and revolutionize our understanding of microbial communities by encompassing both the host and environmental aspects [21]. The metagenomic analysis of fecal microbiomes by Singh et al. [26] indicated that fecal microbiota may be related to broiler performance and suggested the potential use of these tools for improving feed efficiency and weight gain

*Corresponding author

Tel: +82-43-261-3356, Fax: +82-43-261-3224

E-mail: moip@cbu.ac.kr 
via the development of poultry-specific probiotics. The effects of antibiotics or probiotics on the gut microbiome of broilers were also evaluated using metagenomic techniques [7, 27]. Next generation sequencing technologies provide the opportunity to examine the bacterial diversity of broilers and lays the foundation for future studies to assess the fecal or intestinal microbial changes associated with growth performance.

The aims of this study were to describe the microbial communities in feces and the intestinal contents of duodena, jejuna, and ceca harvested from broilers, using the pyrosequencing-based 16S rRNA gene analyses, and to characterize the fecal and gut microbiota of broilers during the rearing period to identify the possible relationships between particular microbial profiles, health, and weight gain.

\section{Materials and Methods}

\section{Poultry farm and birds}

Three commercial broiler farms in Jincheon-gun, one of the largest broiler-growing regions in South Korea, were enrolled in this study. Two farms (farms B and C) were under contract by the same company and received the same feed and bedding materials. The remaining farm (farm A) used different feed and litter. All three farms were provided with a standard corn- and soybean-based commercial feed and did not use any antibiotics, growth promoters, or probiotics during the experiments. The birds were one-day-old Ross-type broiler chicks from commercial hatcheries (farms B and C received their broiler chicks from the same hatchery) and were grown to approximately four to five weeks old. All birds from the three farms were vaccinated using an aerosol spray delivery of the Newcastle disease vaccination at hatching and a water-administered infectious bursal disease vaccination at $12-14$ days old.

\section{Sample characterization}

Two independent experiments were performed. In experiment I, farm-level differences in the fecal microbiomes of three commercial broiler farms (farms A, B, and C) were examined from December 2013 to February 2014. The farms were examined when the chicks were four days old and again at 28 days. The samples consisted of pooled stool collected from different locations (a minimum of 10 sites) at each farm over the broiler-rearing period, using disposable wooden applicators and sterile sampling bags. Upon collection, the samples were kept frozen at $-20^{\circ} \mathrm{C}$, preceding DNA purification.

In experiment II, one commercial broiler flock from farm B was selected to analyze intestinal samples to determine differences in intestinal segments. From February to March 2015 and based on the body weight of the broilers at 31days-old, the top 40 ranked birds were randomly divided into four heavy-weight groups of 10 birds each. The bottomranked 40 birds were randomly divided into four light-weight groups, also of 10 birds each. A one-way analysis of the variance was used to examine the differences in body weight between the heavy and light groups, which were found to be statistically significant at $p<0.01$ (data not shown). Eighty of the 31-day-old Ross-type birds were euthanized and duodenal, jejunal, and cecal sections of their intestines were aseptically collected and homogenized. Homogenates included both intestinal contents and intestinal walls. For each euthanized bird, individual body weight was recorded. All animal handling procedures were conducted according to protocols approved by the institutional animal care and use committee of Chungbuk National University, in Cheongju, South Korea.

\section{DNA extraction}

Total DNA containing the microbial communities was extracted using a QIAamp DNA Stool Mini Kit (Qiagen, USA) according to the manufacturer's instructions. The extracted DNA was used as a template in the polymerase chain reaction (PCR) to amplify the $16 \mathrm{~S}$ rRNA genes.

\section{Pyrosequencing}

The extracted DNA was amplified using barcoded fusion primers targeting the V1-V3 hypervariable regions of $16 \mathrm{~S}$ rRNA genes. The primers contained the 454 adapter, a key sequence (four bases), a barcode sequence (seven-nine bases, only in the reverse primer), a linker (two bases) and the sequence of the universal primers $27 \mathrm{~F}$ and $518 \mathrm{R}$ [3]. The forward primer sequence was 5'-CCTATCCCCTGTGTGCCTTGGCAGTC-TCAG-AC-GAGTTTGATCMTGGCTCAG-3', and the reverse primer sequence was 5'-CCATCTCATCCCTGCGTGTCTCCGAC-TCAG-X-AC-WTTACCGCGGCTGCTGG-3'; the X represents the barcode sequence, which was a unique sequence designed to differentiate sequencing reads from different samples. PCR was performed using a C1000 Touch Thermal Cycler (Bio-Rad Laboratories, USA) with the cycling conditions: An initial denaturation step (5 min at $94^{\circ} \mathrm{C}$ ), followed by 30 cycles of denaturation $\left(30 \mathrm{sec}\right.$ at $\left.94^{\circ} \mathrm{C}\right)$, annealing $\left(45 \mathrm{sec}\right.$ at $\left.55^{\circ} \mathrm{C}\right)$ and extension $\left(90 \mathrm{sec}\right.$ at $\left.72^{\circ} \mathrm{C}\right)$. The amplified PCR products were confirmed by $2 \%$ agarose gel electrophoresis and visualized under the Gel Doc system (Bio-Rad Laboratories). Amplicons were purified with a QIAquick PCR Purification Kit (Qiagen) and quantified using a PicoGreen dsDNA Assay Kit (Invitrogen, USA). The equimolar concentrations of each amplicon, from different samples, were pooled and purified using an AMPure Bead kit (Agencourt Bioscience, USA) and then amplified on sequencing beads by emulsion PCR. Sequencing reactions were performed using a Roche/454 GS Junior System (Roche, Switzerland) following the manufacturer's instructions.

\section{Sequence data analysis}

The analysis of pyrosequenced data was conducted according to the methods used in previous research [4]. Briefly, raw pyrosequencing reads from the fecal and intestinal samples were sorted by sample-specific barcode sequences, the barcoded fusion primers were trimmed, and low quality reads (average quality score $<25$ or read length $<300 \mathrm{bp}$ ) were removed. 
The sequencing results were compared with sequence data deposited in GenBank using the BLAST search program to detect both non-targeting 16S rRNA gene sequences and chimeric sequences from the sequencing reads, and these sequences were filtered out. In this study, strain identification was analyzed using EzTaxon and EZBioCloud database (ChunLab, Korea) [5]. The sequences were clustered into operational taxonomic units (OTUs) at $97 \%$ similarity.

All statistical analyses of the bacterial communities were carried out with CLcommunity software (ChunLab). The Shannon index (diversity index) and Chaol (diversity estimator) were calculated using RDP pyrosequencing pipelines [6].
The OTUs unique to a specific sample were defined with the CD-HIT program [13] at 3\% sequence dissimilarity using Taxon XOR analysis. Principal coordinate analyses (PCoA) were used to compare relationships among the samples using the unweighted UniFrac distance metric [15], and a heat map was used to represent the relative abundance of dominant genera.

\section{Statistical analysis}

The data were expressed as mean $\pm \mathrm{SE}$, and statistical analyses were performed using a statistical analysis package (ver. 22; IBM, USA).

Table 1. The general properties and 16S rRNA gene libraries of each sample in Experiments I and II

\begin{tabular}{|c|c|c|c|c|c|}
\hline Sample ID* & Sampling date $(\mathrm{d})$ & Samples & Number of reads & Number of OTU & Description \\
\hline \multicolumn{6}{|l|}{ Experiment I } \\
\hline $\mathrm{A} 4 \mathrm{~F}$ & 4 & Feces & 6,257 & 1,137 & \multirow{2}{*}{$\begin{array}{l}\text { Pooled fecal sample from } \\
\text { Farm A at } 4 \text { and } 28 \text { days }\end{array}$} \\
\hline $\mathrm{A} 28 \mathrm{~F}$ & 28 & Feces & 6,529 & 1,458 & \\
\hline $\mathrm{B} 4 \mathrm{~F}$ & 4 & Feces & 9,803 & 827 & \multirow{2}{*}{$\begin{array}{l}\text { Pooled fecal sample from } \\
\text { Farm B at } 4 \text { and } 28 \text { days }\end{array}$} \\
\hline $\mathrm{B} 28 \mathrm{~F}$ & 28 & Feces & 4,385 & 815 & \\
\hline $\mathrm{C} 4 \mathrm{~F}$ & 4 & Feces & 6,319 & 828 & \multirow{2}{*}{$\begin{array}{l}\text { Pooled fecal sample from } \\
\text { Farm } \mathrm{C} \text { at } 4 \text { and } 28 \text { days }\end{array}$} \\
\hline $\mathrm{C} 28 \mathrm{~F}$ & 28 & Feces & 5,312 & 1,017 & \\
\hline \multicolumn{6}{|l|}{ Experiment II } \\
\hline H1D & \multirow{3}{*}{31} & Duodenum & 19,507 & 1,843 & \multirow{3}{*}{$\begin{array}{l}\text { Pooled intestinal sample } \\
\text { from heavy group } 1\end{array}$} \\
\hline $\mathrm{H} 1 \mathrm{~J}$ & & Jejunum & 20,773 & 1,578 & \\
\hline $\mathrm{H} 1 \mathrm{C}$ & & Cecum & 52,06 & 1,212 & \\
\hline $\mathrm{H} 2 \mathrm{D}$ & \multirow{3}{*}{31} & Duodenum & 16,973 & 1,957 & \multirow{3}{*}{$\begin{array}{l}\text { Pooled intestinal sample } \\
\text { from heavy group } 2\end{array}$} \\
\hline $\mathrm{H} 2 \mathrm{~J}$ & & Jejunum & 19,424 & 1,628 & \\
\hline $\mathrm{H} 2 \mathrm{C}$ & & Cecum & 6,425 & 1,486 & \\
\hline $\mathrm{H} 3 \mathrm{D}$ & \multirow{3}{*}{31} & Duodenum & 28,133 & 2,613 & \multirow{3}{*}{$\begin{array}{l}\text { Pooled intestinal sample } \\
\text { from heavy group } 3\end{array}$} \\
\hline $\mathrm{H} 3 \mathrm{~J}$ & & Jejunum & 29,562 & 2,084 & \\
\hline $\mathrm{H} 3 \mathrm{C}$ & & Cecum & 6,718 & 1,517 & \\
\hline H4D & \multirow{3}{*}{31} & Duodenum & 26,140 & 2,325 & \multirow{3}{*}{$\begin{array}{l}\text { Pooled intestinal sample } \\
\text { from heavy group } 4\end{array}$} \\
\hline $\mathrm{H} 4 \mathrm{~J}$ & & Jejunum & 26,651 & 2,141 & \\
\hline $\mathrm{H} 4 \mathrm{C}$ & & Cecum & 6,141 & 1,523 & \\
\hline L1D & \multirow{3}{*}{31} & Duodenum & 16,556 & 2,787 & \multirow{3}{*}{$\begin{array}{l}\text { Pooled intestinal sample } \\
\text { from light group } 1\end{array}$} \\
\hline L1J & & Jejunum & 16,457 & 2,508 & \\
\hline $\mathrm{L} 1 \mathrm{C}$ & & Cecum & 5,939 & 1,435 & \\
\hline L2D & \multirow{3}{*}{31} & Duodenum & 11,623 & 2,385 & \multirow{3}{*}{$\begin{array}{l}\text { Pooled intestinal sample } \\
\text { from light group } 2\end{array}$} \\
\hline L2J & & Jejunum & 11,918 & 2,220 & \\
\hline L2C & & Cecum & 7,678 & 1,530 & \\
\hline L3D & \multirow{3}{*}{31} & Duodenum & 23,698 & 3,125 & \multirow{3}{*}{$\begin{array}{l}\text { Pooled intestinal sample } \\
\text { from light group } 3\end{array}$} \\
\hline L3J & & Jejunum & 18,754 & 2,460 & \\
\hline L3C & & Cecum & 6,010 & 1,404 & \\
\hline L4D & \multirow{3}{*}{31} & Duodenum & 16,514 & 2,668 & \multirow{3}{*}{$\begin{array}{l}\text { Pooled intestinal sample } \\
\text { from light group } 4\end{array}$} \\
\hline L4J & & Jejunum & 4,185 & 971 & \\
\hline $\mathrm{L} 4 \mathrm{C}$ & & Cecum & 7,539 & 1,459 & \\
\hline Total & & & 397,129 & 52,941 & \\
\hline
\end{tabular}

OUT, operational taxonomic unit. *Sample identification number. Thirty samples were analyzed including pooled fecal samples for interfarm comparisons (Experiment I) and individual intestinal samples for intra-farm comparisons (Experiment II). 


\section{Results}

\section{Information of pyrosequencing data}

In this study, the microbial compositions and changes in bacterial profiles of feces and intestinal contents during the rearing period of broilers were investigated via the pyrosequencing of $16 \mathrm{~S}$ rRNA genes. Thirty samples were analyzed, including pooled fecal samples for inter-farm comparisons (Experiment I) and intestinal samples of heavy and light birds for intra-farm comparisons (Experiment II). From these samples, 397,129 reads were generated and rarefied to a total of 52,941 OTUs. The information extracted from the analyzed samples is summarized in Table 1.

\section{Microbial composition in fecal samples from broiler} farms

The mean prevalence of each microbial phylum from the fecal samples taken at four and 28 days old are presented in Figure 1. In farm A, Firmicutes was the major phylum, followed by Proteobacteria and Bacteroidetes. In contrast, Proteobacteria was detected as the major phylum in both farms $\mathrm{B}$ and $\mathrm{C}$, followed by Firmicutes. At a lower taxonomic level, genus-level differences were reported among the three farms at four days (Fig. 2A) and 28 days (Fig. 2B). The most abundant bacteria found in fecal samples from the fourth consisted of the Escherichia genus. In the four-day fecal samples from farm A, genera such as Clostridium, Proteus, and Lactobacillus were found in higher proportions, compared to the fecal samples from farms B and C. In the 28-day samples, a high abundance of Alistipes was found in feces from farm A. In the samples from farms B and C, Lactobacillus genus was increased in the 28-day fecal samples compared to the four-day samples. The bacterial population increased and changed over time, consisting of a few types of bacteria, mostly the coliforms Lactobacillus, Clostridium, Bacteroides, and Proteus, which were later replaced by a more complex and diverse microbiome.

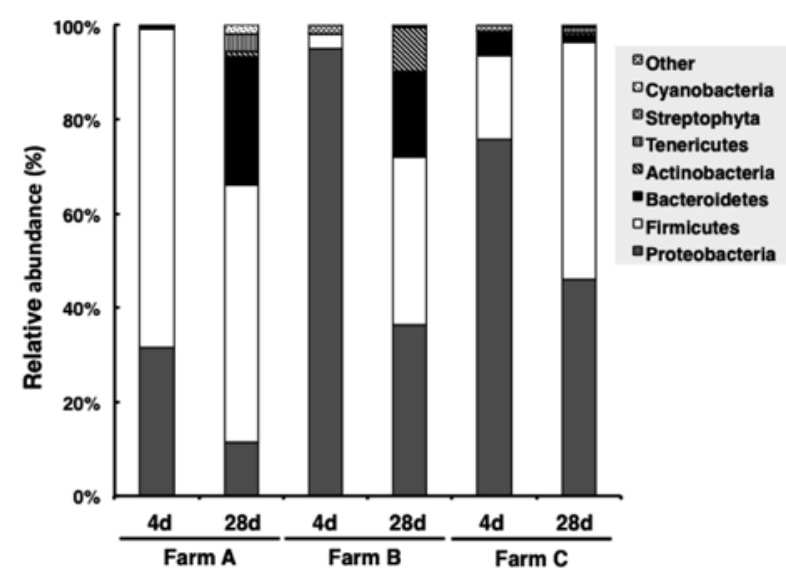

Fig. 1. Relative abundance of major phylum in fecal samples from broiler farms at 4-days old (4d) and 28-days old (28d).
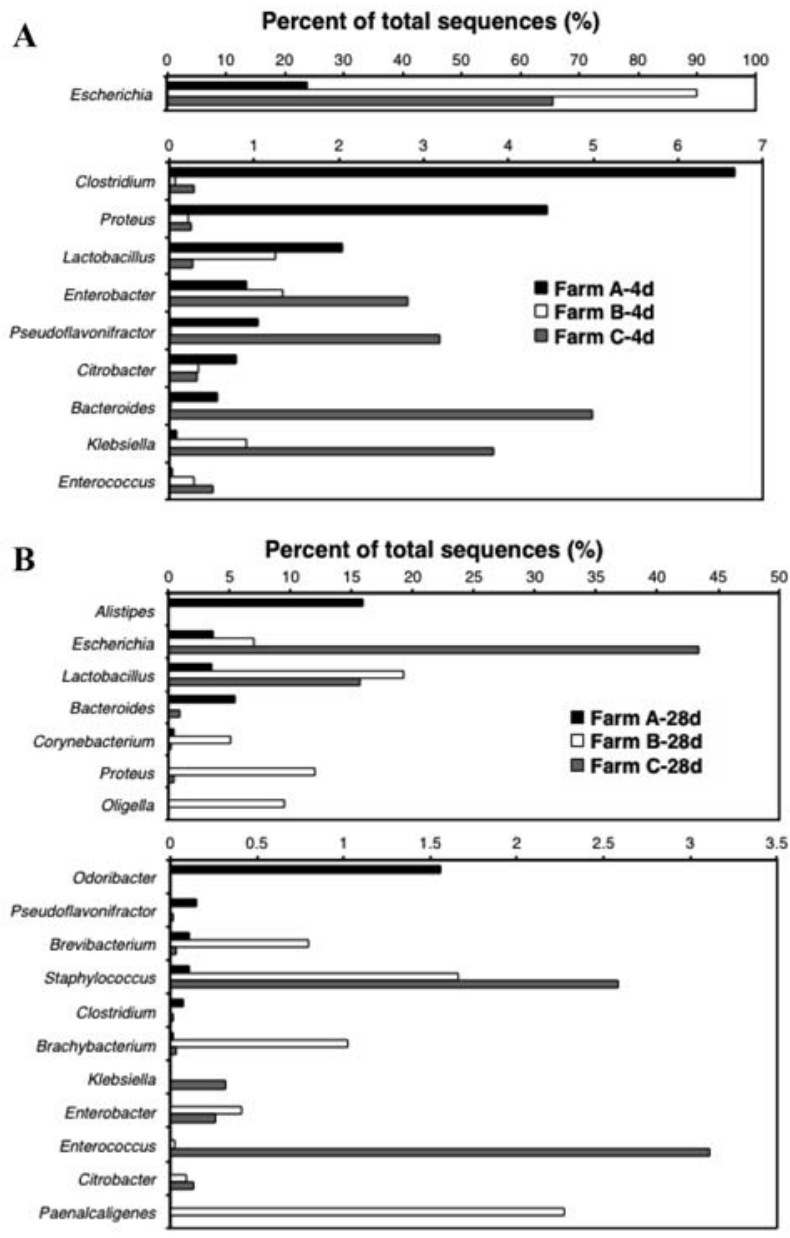

Fig. 2. Relative abundance of bacterial genera in fecal samples from broiler farms at 4-days old (A, 4d) and 28-days old (B, 28d).

Microbial diversity and farm-specific OTUs in fecal samples from broiler farms

As shown in Figure 3A, regardless of the farm, Shannon index and Chaol gradually increased from four to 28 days in all three commercial broiler farms, suggesting that the bacterial diversity of each sample became greater as the animals got older. The microbial profiles of the three commercial broiler farms were represented using PCoA (Fig. 3B). PCoA plots categorized fecal samples according to the farm, and illustrated the microbial succession from four to 28 days, which were found in all three farms. The early microbial profiles from the three broiler farms revealed differences according to farm environments and geographic location. Farm A presented the highest richness and diversity indices of fecal microbiota, and a large number of OTUs unique to itself. Lactobacillus, Blautia, and Roseburia genus at four days old and Alistipes and Sutterella at 28 days old were identifiable at the genus level.

Microbial composition in the intestinal samples from the heavy and light birds

The differences in the composition of the microbial commu- 
nity from the intestinal samples of the heavy and light groups at the phylum level are shown in Figure 4. The duodenal and

\section{A}

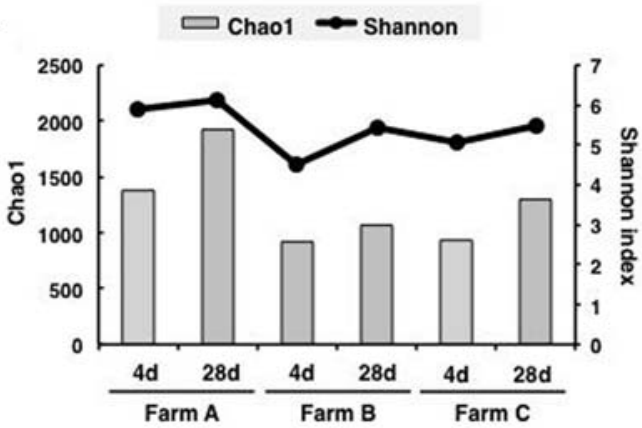

B

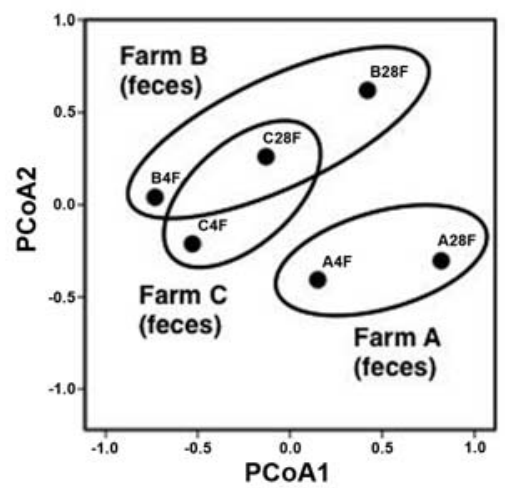

Fig. 3. Microbial richness and diversity $(\mathrm{A})$ and principal coordinate analysis (PCoA1 and PCoA2) of dataset (B) in fecal samples from 3 broiler farms (A, B and C) at 4-days old (4d) and 28days old (28d). These figures (B) illustrate microbial transitions. jejunal samples from the heavy groups revealed a predominance of Firmicutes, while the bacterial communities in the duodenum and jejunum from the light groups contained greater proportions of Firmicutes, Proteobacteria, and Bacteroidetes, and smaller proportions of Tenericutes and Actinobacteria.

As illustrated in the heat map of Figure 5, the results revealed that the microbial communities in the intestinal tract varied between the heavy and light groups. At the lower phylogenetic levels, Lactobacillus genus was predominantly detected in the duodenum and jejunum, while Alistipes and Sutterella were dominant in the cecum. Compared to the heavy groups, the small intestines from the light groups had a relatively lower abundance of Lactobacillus and higher abundances of Escherichia, Bacteroides, Clostridium, and Eimeria. In the ceca harvested from the light groups, higher percentages of Bacteroides were observed than in the ceca harvested from the heavy groups.

Microbial diversity and group-specific OTUs in intestinal samples from the heavy and light birds

The PCoA plot representations of the intestinal segments from the heavy and light groups (Fig. 6) were separated into different clusters based on the locations in the gut. In addition, the inter-individual variations within samples from the light groups were greater than those within samples from heavy groups. Although the microbial communities found in the duodenum and jejunum were dispersed sporadically compared to those in the cecum, small intestinal segments were generally clustered in a group-specific manner, and cecal segments were clustered in a segment-specific manner. It was demonstrated that both the heavy and light groups repre-

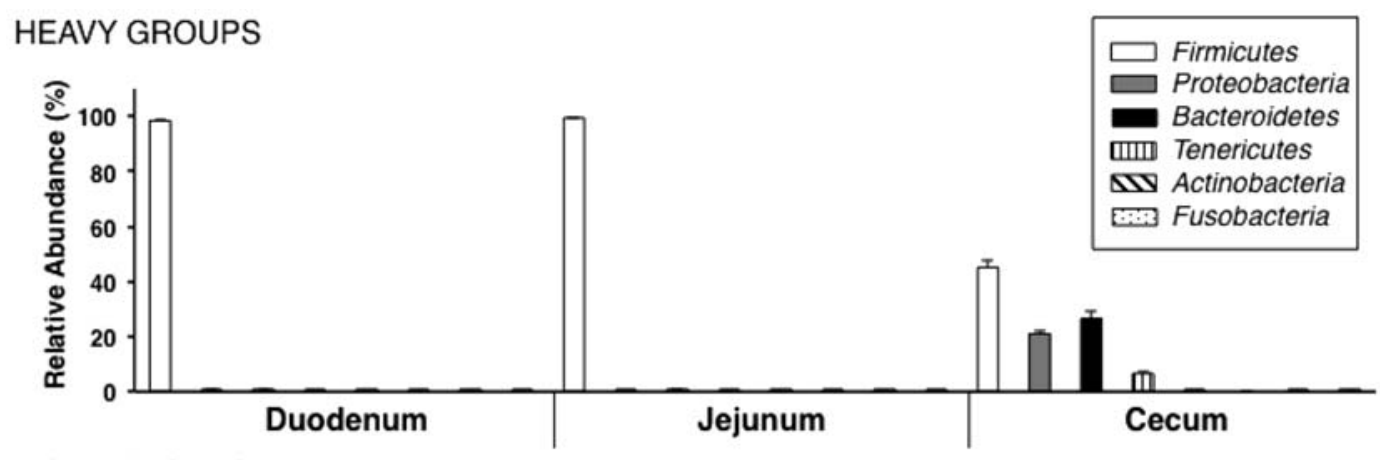

\section{LIGHT GROUPS}

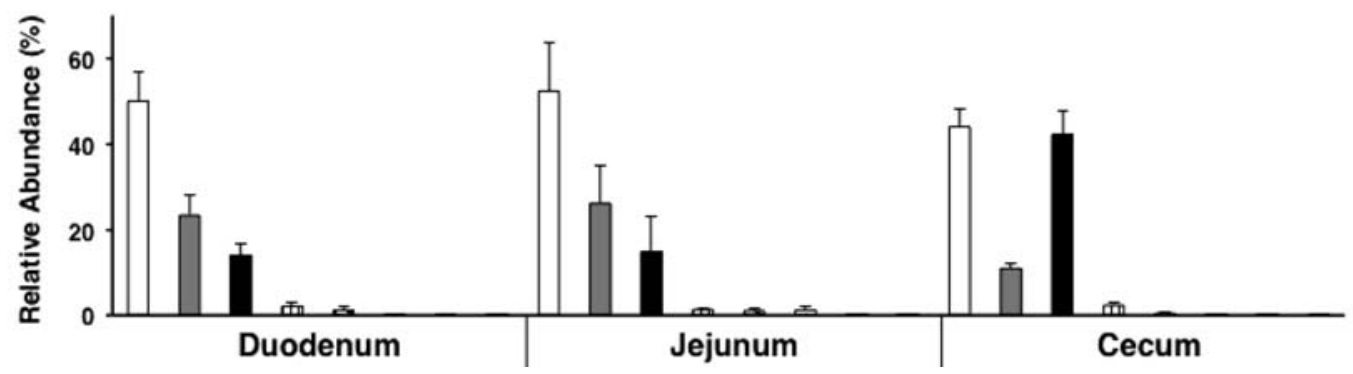

Fig. 4. Relative abundance of major phylum in intestinal samples from the heavy and light groups. 


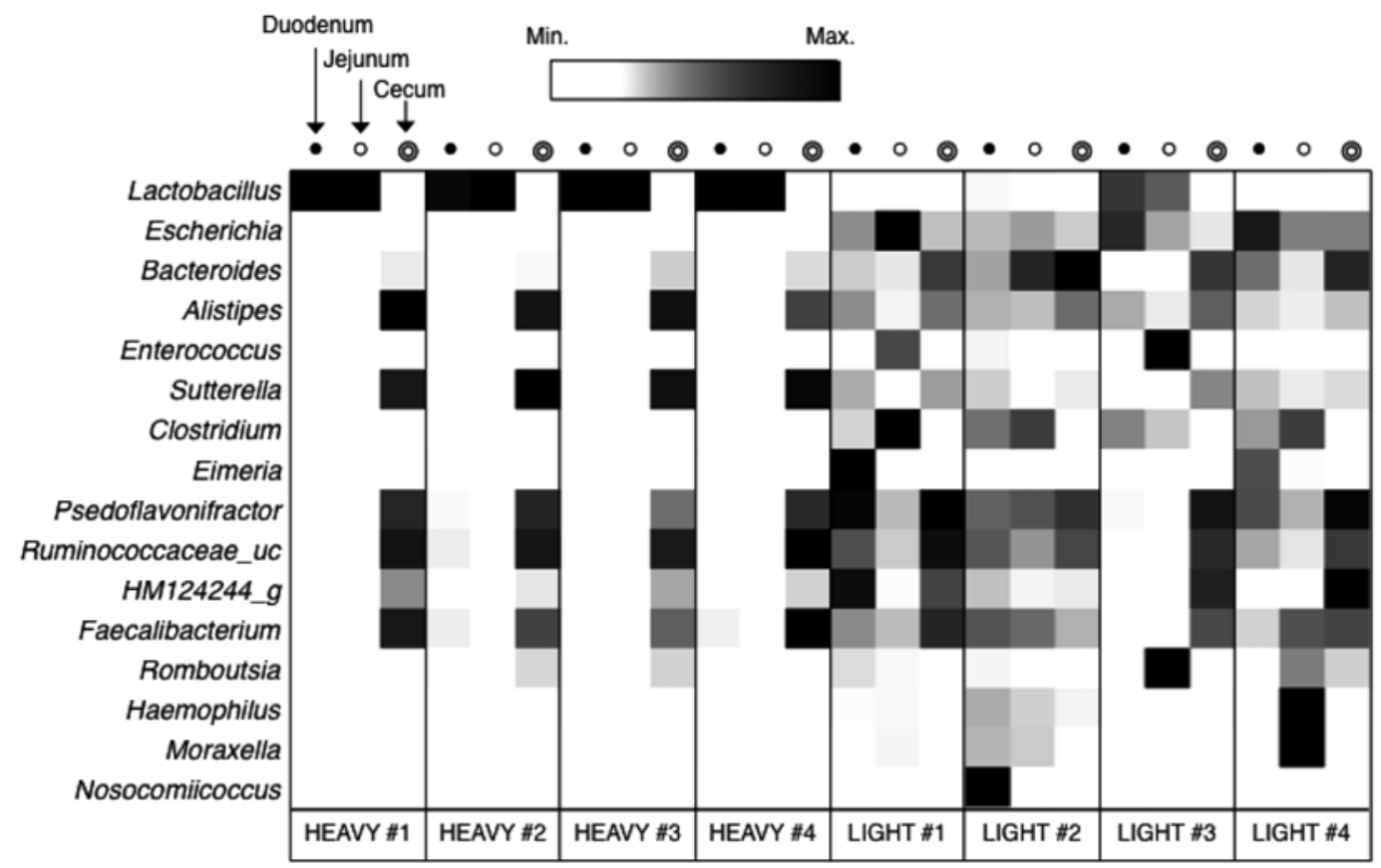

Fig. 5. Relative abundance of bacterial genera in intestinal samples from the heavy and light groups. Each column in the heat map showed duodenum (O), jejunum $(\bigcirc)$, and cecum (O), respectively. The intensity of each panel is proportional to the abundance of each genus.

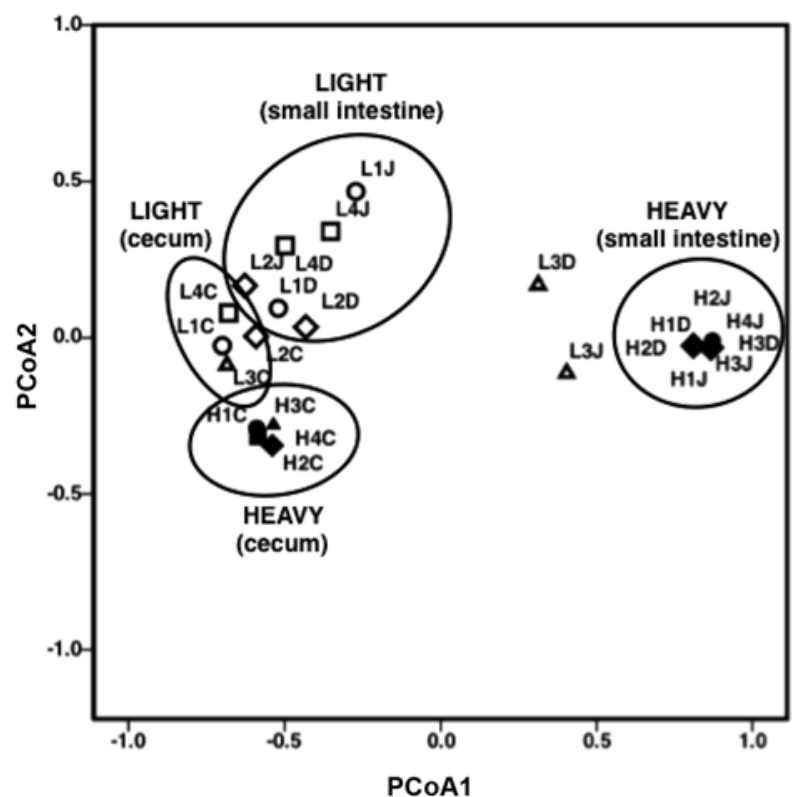

Fig. 6. Principal coordinate analysis (PCoA1 and PCoA2) in intestinal samples (small intestine and cecum) from the heavy and light groups. The letters in the symbols are the unique numbers of the samples as described in Table 1.

sented body weight-specific clustering with differences in the gut microbial profiles, particularly in the small intestine.

Taxon XOR analysis by CD-HIT function detected several OTUs unique to the light groups. Clostridium perfringens was mainly detected in all the intestinal segments of the light groups (data not shown). Other OTUs, which are members of the Clostridia class, were more abundant in the light groups and may have contributed to the observed differences between body weights. Both the increase in Clostridium and decrease in Lactobacillus, were found in the intestinal samples of the light groups compared to those of the heavy groups. Also, there were no specific pathological lesions in the intestinal tissues from the light groups.

\section{Discussion}

Promoting growth performance of broilers has been one of the most important aims in the poultry industry. Considerable improvements have occurred, such as shorter market age and higher feed conversion ratios, achieved through genetic selection [29]. A number of studies are still on going to elucidate the microbial community's involvement in growth performance. This study, using pyrosequencing of 16S rRNA, investigated the degree of microbial diversity and changes in bacterial profiles during the rearing period of broilers.

The development of gastrointestinal microflora is believed to begin immediately after hatching. Environment has a major influence on the formation of a bird's gut microbiome. As seen in our results from Experiment I, the early microbial profiles from three broiler farms revealed differences according to farm environments and geographic location, and the results are in accordance with those from other study [40]. The chicks from both farms $\mathrm{B}$ and $\mathrm{C}$, which were raised in similar environment that included the same feed, bedding 
materials, handlers and transports, revealed similar microbial profiles at early ages. The considerably different environmental conditions between farms $\mathrm{B}$ and $\mathrm{C}$ resulted from construction systems (i.e., modern vs. old-fashioned housing systems), which may have influenced on these differences in the fecal microbial communities from each farm; however, significant differences between farms $\mathrm{B}$ and $\mathrm{C}$ could not be found

Overall, the fecal microbial profiles from three broiler farms were consistently maintained and shifted only slightly, and the composition of fecal microbiome from commercial broiler farms was similar to results reported in previous studies (e.g., Firmicutes or Proteobacteria predominating, followed by Bacteroidetes) [37]. The bacterial population increased and changed over time, consisting of a few types of bacteria, mostly the coliforms, Lactobacillus, Bifidobacterium, and Clostridia, which was later replaced by a more complex and diverse microbiome. Farm A had the highest richness, diversity, and a large number of unique farm-specific OTUs. Lactobacillus, Blautia, and Roseburia species at four days old and Alistipes and Sutterella at 28 days old were identifiable to the genus level, and some of them were known to potentially be performance-related phylotypes [33]. Among these bacteria, Alistipes finegoldii has been known to play a role in metabolism of $\mathrm{N}$-glycan in a similar way as the intestinal anaerobic bacteria in the Bacteroidetes phylum does [20].

The fecal microbiome is known to be directly influenced by the emptying of different intestinal segments, greatly different at various time points [24], and offer a limited representation of the gut microflora [30]. Some crucial phylotypes from fecal samples should not be neglected. In Experiment I, a notable correlation between the fecal microbiome and body performance could not be found due to the limited number of samples. In spite of the limitations, pyrosequencing data of fecal microbiome established the basis for a potential monitoring system to improve feed efficiency and validation of the presence of performance-related bacteria, which could lead to the use of farm-specific probiotics.

As shown in Figure 3A, regardless of the farm, Shannon index and Chaol gradually increased from four to 28 days in all three commercial broiler farms, suggesting that the bacterial diversity of each sample became greater as the animals got older. The microbial profiles of the three commercial broiler farms were represented using PCoA. PCoA plots categorized fecal samples according to the farm, and illustrated the microbial succession from four to 28 days, which were found in all three farms. The early microbial profiles from the three broiler farms revealed differences according to farm environments and geographic location, and the results are in accordance with those from another study [40].

The microbial colonization from different segments of the chicken GIT are quite different and it has been implicated that there is spatial heterogeneity [36]. Because it is difficult to define typical bacteria profiles for segments of the GIT and to minimize the individual variations, the intestinal samples were pooled in Experiment II. Choi et al. [2] investi- gated similarities in gut microbiome and found that microbial communities in the upper GIT, such as the crop, gizzard, duodenum, and jejunum, showed some similarities, although each had a specific bacterial profile. The microbiome in cecum and the large intestines were shown to be very different, when compared to that in other intestinal segments.

As seen in a study by Gong et al. [11], cecum showed the highest bacterial diversity index among the intestinal sections. This pattern was found only in the heavy groups, and the diversity indices of the small intestines were higher in light groups, which suggest that heavy-weight individuals with abnormal energy input showed significant reduced diversity in gut microflora. This is also seen in the gut microbiome of obese humans [35]. Bacterial richness in intestinal samples revealed no significant differences between the heavy and light groups, which means that variability in microbial communities among individual intestinal samples have caused insignificant results in this study.

According to comparisons of microbial populations from intestinal samples from the heavy and light groups, duodenal and jejunal metagenomic profiles were premominantly made up of Firmicutes (comprised of Lactobacillus crispatus, Lactobacillus johnsonii, Lactobacillus taiwanensis, and Lactobacillus salivarius), which in agreements with studies that used conventional culture methods [23]. In addition, low population of Escherichia coli, Enterococcus, Feacalibacterium, Clostridium, and Bacteroides species were observed in the small intestine of both the heavy and light groups, involving utilization of polysaccharides, nutrient absorption, and development of the intestinal immune system [17]. In chickens, ceca are an important part of fermentation, water absorption, and nutrient transport and absorption, and involved in both maintaining the host's health and growth performance [21]. The cecal microbial communities showed relatively higher proportions of bacterial phylum, such as Bacteroidetes and Proteobacteria, and a higher abundance of Alistipes, Sutterella, and Bacteroides species compared to those of the small intestines. The relationship between feed efficiency and gut microbiome is more pronounced in the proximal intestine at early ages and in distal parts as chicken ages [8]. Some phylum, such as Firmicutes, Bacteroidetes, and Fusobacteria, were associated with high feed conversion ratios and have a positive impact on the energy yield from the feed and body weight gain [26, 35]. According to previous studies, several probiotic candidates associated with growth performance were found, including Lactobacillus cripatus, Lactobacillus aviaries, Lactobacillus reuteri, and Weissella species, in Firmicutes phylum [19, 31, 34].

Most researches, focusing on chicken GIT, emphasize the importance of cecum as a critical site associated with body performance and major pathogen reservoirs, when compared to other intestinal segments [7, 27]. Although several studies reported an unclear relationship between body performance and jejunal microbiome [34], this study found substantial discrepancies in the duodenal and jejunal bacterial profiles 
between the light and heavy groups. It is possible that poor health, which was induced by other factors, caused disturbances in gut microbiome of the light groups. Even though it is uncertain what changes occurred previously, this could suggest that microbial communities are related and instantly reflected the health status of the host. Unlike the heavy group, microbial profiles were constantly maintained from the duodenum to the cecum in the light groups. This tendency might have occurred because of retrograde movement of intestinal contents in the chicken GIT, which influence microbial communities both up and down stream [28].

The bacterial pathogens within fecal or intestinal microbiome are an important part of both animal and public health. In this study, the major zoonotic pathogens, such as Salmonella enteritidis and Campylobacter species, could not be identified. Clostridium perfringens is a well-known pathogen that induces necrotic enteritis in poultry and was detected at high levels in the gut microbiome of the light groups. For long time, antibiotic growth promoters have been added to feed to improve growth efficiency and control bacterial agents, such as Clostridium perfringens. Effects of feed additives on the gut microbiome are an increase in Clostridia and a reduction of Lactobacillus species were reduced [16]. Similar patterns for microbial shifts were found in the intestinal samples of the light groups. It seems that healthy gut microbiome from the heavy groups could reduce overgrowth of Clostridium perfringens, by resisting changes in bacterial composition [31]. There were no specific pathological lesions in the intestinal tissues from the light groups. In other words, although the presence of Clostridium perfringens was found in light-weight chickens' microbiomes at detectable levels, it does not always indicate a diseased state and might have a negative impact on growth efficiency.

Exploring the balance and dynamics of the microbial community is necessary to develop strategies to improve growth performance and to identify adequate feed additives, which influence a well-balanced microbiome. Besides understanding the behaviors of the gut microbiome, it is also important to understand a normal state of poultry flocks. In future research, microbiome dynamics and relationship with body performance can be determined using meaningful data, which can be done by increasing sampling numbers and time points. The accumulation of data about bacterial communities from various types of samples allows an understanding of the core microbiome to improve the growth efficiency of broilers.

\section{Acknowledgments}

This work was supported by Korea Institute of Planning and Evaluation for Technology in Food, Agriculture, Forestry and Fisheries (IPET) through Animal Disease Management Technology Development Program, funded by Ministry of Agriculture, Food and Rural Affairs (MAFRA) (315045032SB010) and was supported by a 2014 research grant from Chungbuk National University.

\section{References}

1. Aarestrup FM, Agerso Y, Gerner-Smidt P, Madsen M, Jensen LB. Comparison of antimicrobial resistance phenotypes and resistance genes in Enterococcus faecalis and Enterococcus faecium from humans in the community, broilers, and pigs in Denmark. Diagn Microbiol Infect Dis 2000, 37, 127-137.

2. Choi JH, Kim GB, Cha CJ. Spatial heterogeneity and stability of bacterial community in the gastrointestinal tracts of broiler chickens. Poult Sci 2014, 93, 1942-1950.

3. Chun J, Goodfellow M. A phylogenetic analysis of the genus Nocardia with 16S rRNA gene sequences. Int J Syst Bacteriol 1995, 45, 240-245.

4. Chun J, Kim KY, Lee JH, Choi Y. The analysis of oral microbial communities of wild-type and Toll-like receptor 2deficient mice using a 454 GS FLX Titanium pyrosequencer. BMC Microbiol 2010, 10, 101.

5. Chun J, Lee JH, Jung Y, Kim M, Kim S, Kim BK, Lim YW. EzTaxon: a web-based tool for the identification of prokaryotes based on $16 \mathrm{~S}$ ribosomal RNA gene sequences. Int J Syst Evol Microbiol 2007, 57, 2259-2261.

6. Cole JR, Wang Q, Fish JA, Chai B, McGarrell DM, Sun Y, Brown CT, Porras-Alfaro A, Kuske CR, Tiedje JM. Ribosomal database project: data and tools for high throughput rRNA analysis. Nucleic Acids Res 2014, 42, D633-642.

7. Danzeisen JL, Kim HB, Isaacson RE, Tu ZJ, Johnson TJ. Modulations of the chicken cecal microbiome and metagenome in response to anticoccidial and growth promoter treatment. PLoS One 2011, 6, e27949.

8. de Lange LL, Wijtten PJA. Microbial profiles of the gastro-intestinal tract of broilers and its relation to feed efficiency. In: 21st Annual Australian Poultry Science Symposium; 1-3 February, Sydney, Australia.

9. Emborg HD, Andersen JS, Seyfarth AM, Wegener HC. Relations between the consumption of antimicrobial growth promoters and the occurrence of resistance among Enterococcus faecium isolated from broilers. Epidemiol Infect 2004, 132, 95105.

10. Fernando PS, Rose SP, Mackenzie AM, Silva SSP. Effect of diets containing potato protein or soya bean meal on the incidence of spontaneously-occurring subclinical necrotic enteritis and the physiological response in broiler chickens. $\mathrm{Br}$ Poult Sci 2011, 52, 106-114.

11. Gong J, Forster RJ, Yu H, Chambers JR, Wheatcroft R, Sabour PM, Chen S. Molecular analysis of bacterial populations in the ileum of broiler chickens and comparison with bacteria in the cecum. FEMS Microbiol Ecol 2002, 41, 171-179.

12. Hughes RJ. An integrated approach to understanding gut function and gut health of chickens. Asia Pac J Clin Nutr 2005, 14 (Suppl), S27.

13. Li W, Godzik A. Cd-hit: a fast program for clustering and comparing large sets of protein or nucleotide sequences. Bioinformatics 2006, 22, 1658-1659.

14. Long JR. Necrotic enteritis in broiler chickens I. A review of the literature and the prevalence of the disease in Ontario. Can J Comp Med 1973, 37, 302-308.

15. Lozupone C, Knight R. UniFrac: a new phylogenetic method for comparing microbial communities. Appl Environ Microbiol 2005, 71, 8228-8235. 
16. Lu J, Hofacre C, Smith F, Lee MD. Effects of feed additives on the development on the ileal bacterial community of the broiler chicken. Animal 2008, 2, 669-676.

17. Lu J, Idris U, Harmon B, Hofacre C, Maurer JJ, Lee MD. Diversity and succession of the intestinal bacterial community of the maturing broiler chicken. Appl Environ Microbiol 2003, 69, 6816-6824.

18. Miles RD, Butcher GD, Henry PR, Littell RC. Effect of antibiotic growth promoters on broiler performance, intestinal growth parameters, and quantitative morphology. Poult Sci 2006, 85, 476-485.

19. Nakphaichit M, Thanomwongwattana S, Phraephaisarn C, Sakamoto N, Keawsompong S, Nakayama J, Nitisinprasert S. The effect of including Lactobacillus reuteri KUB-AC5 during post-hatch feeding on the growth and ileum microbiota of broiler chickens. Poult Sci 2011, 90, 2753-2765.

20. Nihira T, Suzuki E, Kitaoka M, Nishimoto M, Ohtsubo K, Nakai H. Discovery of $\beta-1,4-D-m a n n o s y l-N$-acetyl-Dglucosamine phosphorylase involved in the metabolism of $\mathrm{N}$ glycans. J Biol Chem 2013, 288, 27366-27374.

21. Oakley BB, Lillehoj HS, Kogut MH, Kim WK, Maurer JJ, Pedroso A, Lee MD, Collett SR, Johnson TJ, Cox NA. The chicken gastrointestinal microbiome. FEMS Microbiol Lett 2014, 360, 100-112.

22. Salanitro JP, Blake IG, Muirehead PA, Maglio M, Goodman JR. Bacteria isolated from the duodenum, ileum, and cecum of young chicks. Appl Environ Microbiol 1978, 35, 782-790.

23. Salanitro JP, Fairchilds IG, Zgornicki YD. Isolation, culture characteristics, and identification of anaerobic bacteria from the chicken cecum. Appl Microbiol 1974, 27, 678-687.

24. Sekelja M, Rud I, Knutsen S, Denstadli V, Westereng B, Nas T, Rudi K. Abrupt temporal fluctuations in the chicken fecal microbiota are explained by its gastrointestinal origin. Appl Environ Microbiol 2012, 78, 2941-2948.

25. Shira EB, Sklan D, Friedman A. Impaired immune responses in broiler hatchling hindgut following delayed access to feed. Vet Immunol Immunopathol 2005, 105, 33-45.

26. Singh KM, Shah T, Deshpande S, Jakhesara SJ, Koringa PG, Rank DN, Joshi CG. High through put 16S rRNA gene-based pyrosequencing analysis of the fecal microbiota of high FCR and low FCR broiler growers. Mol Biol Rep 2012, 39, 10595-10602.

27. Singh P, Karimi A, Devendra K, Waldroup PW, Cho KK, Kwon YM. Influence of penicillin on microbial diversity of the cecal microbiota in broiler chickens. Poult Sci 2013, 92, 272-276.

28. Sklan D, Shachaf B, Baron J, Hurwitz S. Retrograde movement of digesta in the duodenum of the chick: extent, frequency, and nutritional implications. J Nutr 1978, 108, 1485-1490

29. Stanley D, Denman SE, Hughes RJ, Geier MS, Crowley TM, Chen H, Haring VR, Moore RJ. Intestinal microbiota associated with differential feed conversion efficiency in chickens. Appl Microbiol Biotechnol 2012, 96, 1361-1369.

30. Stanley D, Hughes RJ, Moore RJ. Microbiota of the chicken gastrointestinal tract: influence on health, productivity and disease. Appl Microbiol Biotechnol 2014, 98, 4301-4310.

31. Stanley D, Keyburn AL, Denman SE, Moore RJ. Changes in the caecal microflora of chickens following Clostridium perfringens challenge to induce necrotic enteritis. Vet Microbiol 2012, 159, 155-162.

32. Timbermont L, Haesebrouck F, Ducatelle R, Van Immerseel F. Necrotic enteritis in broilers: an updated review on the pathogenesis. Avian Pathol 2011, 40, 341-347.

33. Torok VA, Hughes RJ, Mikkelsen LL, Perez-Maldonado R, Balding K, MacAlpine R, Percy NJ, Ophel-Keller K. Identification and characterization of potential performancerelated gut microbiotas in broiler chickens across various feeding trials. Appl Environ Microbiol 2011, 77, 5868-5878.

34. Torok VA, Ophel-Keller K, Loo M, Hughes RJ. Application of methods for identifying broiler chicken gut bacterial species linked with increased energy metabolism. Appl Environ Microbiol 2008, 74, 783-791.

35. Turnbaugh PJ, Ley RE, Mahowald MA, Magrini V, Mardis ER, Gordon JI. An obesity-associated gut microbiome with increased capacity for energy harvest. Nature 2006, 444, 10271131.

36. van der Wielen PWJJ, Keuzenkamp DA, Lipman LJA, van Knapen F, Biesterveld S. Spatial and temporal variation of the intestinal bacterial community in commercially raised broiler chickens during growth. Microb Ecol 2002, 44, 286293.

37. Videnska P, Rahman MM, Faldynova M, Babak V, Matulova ME, Prukner-Radovcic E, Krizek I, SmoleMozina S, Kovac J, Szmolka A, Nagy B, Sedlar K, Cejkova D, Rychlik I. Characterization of egg laying hen and broiler fecal microbiota in poultry farms in Croatia, Czech Republic, Hungary and Slovenia. PLoS One 2014, 9, e110076.

38. Wei S, Morrison M, Yu Z. Bacterial census of poultry intestinal microbiome. Poult Sci 2013, 92, 671-683.

39. Yegani M, Korver D. Factors affecting intestinal health in poultry. Poult Sci 2008, 87, 2052-2063.

40. Yin Y, Lei F, Zhu L, Li S, Wu Z, Zhang R, Gao GF, Zhu B, Wang X. Exposure of different bacterial inocula to newborn chicken affects gut microbiota development and ileum gene expression. ISME J 2010, 4, 367-376. 